\title{
Medical devices in Sub-Saharan Africa: optimal assistance via a computerized maintenance management system (CMMS) in Benin
}

\author{
Daton Medenou ${ }^{1}$ - Latif A. Fagbemi ${ }^{1}$ - Roland C. Houessouvo ${ }^{1}$ - Thierry R. Jossou ${ }^{1}$ • Mêtowanou H. Ahouandjinou ${ }^{1}$. \\ Davide Piaggio $^{2}$ • Chams-Deen A. Kinnouezan ${ }^{1} \cdot$ Gabriel A. Monteiro ${ }^{1} \cdot$ Moubarack A. Y. Idrissou $^{1} \cdot$ Ernesto ladanza $^{3}$. \\ Leandro Pecchia ${ }^{2}$ (i)
}

Received: 14 August 2018 / Accepted: 29 November 2018 / Published online: 19 March 2019

(C) The Author(s) 2019

\begin{abstract}
In limited resources settings, Health Technology Management (HTM) presents specific challenges, which significantly differ from those faced in higher income settings. In Sub-Saharan Africa (SSA), HTM requires holistic approaches based on reliable information on medical devices operationalized in local medical locations, which may differ significantly from the USA or European ones. Computerized Maintenance Management System (CMMS) tools offer unprecedented opportunities to optimize medical device organization and management in SSA. Nonetheless, CMMS for SSA should be designed to meet real local needs and facing local economic and organizational constraints. This paper describes the results of a project aimed to design and deploy a context-driven CMMS tool, called "GMaint-KM Benin", which was designed, developed, tested, validated and deployed in the Department of Biomedical Engineering, Ecole Polytechnique d'Abomey-Calavi, University of Abomey-Calavi, Benin. The methods followed to design the CMMS, was inspired by closed-loop control theory. It is based on the idea that an effective CMMS could have been designed using as reference international standards and best practices, while controlling and optimizing CMMS inputs and outputs basing upon information and data measured in Beninese medical locations, following a closed-loop feedback control strategy. The tool is currently fully functional in three Beninese hospitals. The successful method used to design this tool represents a best-practice of optimal co-design for Sub-Saharan Africa, which could be adapted and reused in similar projects aiming to design and deploy health technologies for low-income settings. In perspective, the project aims to enhance "GMaint-KM Benin" and scale it up at the national level. The adoption of such a tool could represent an effective base for comparative studies among African countries.
\end{abstract}

Keywords Medical device · Health technology management · Clinical engineering · Africa · Low-income countries (LIC) . Computerized maintenance management system

\section{Introduction}

Benin is a West African country with an estimated population of 11.49 million [1]. Relevant data (i.e., Area, Median age,

Leandro Pecchia

L.Pecchia@warwick.ac.uk

1 Department of Biomedical Engineering, Ecole Polytechnique d'Abomey-Calavi, University of Abomey-Calavi, 01 BP 2009, Cotonou, Benin

2 School of Engineering, University of Warwick, Coventry CV4 7AL, UK

3 Department of Information Engineering, Università degli Studi di Firenze, Florence, Italy
Ratio of Physician per population and annual gross domestic product (GDP)) regarding Benin and other countries (i.e., Europe, USA, UK and Italy) can be found in Table 1. It is worth noticing that the population of Benin is young and fastgrowing (doubled since the '90s) compared with the other states. However, in 2016, the ratio of physicians per population was sensibly lower if compared to European countries or the USA (2.57 in 2014) [2]. In order to give a comparator, the WHO estimated that each country should have at least 2.5 medical staff (physicians, nurses and midwives) per 1000 people, to provide adequate coverage with primary care interventions [3]. In 2015, the annual gross domestic product (GDP) per person was half of the average GDP for Sub-Saharan Africa, and significantly lower than Europe or the USA [4]. Benin is organized in 12 administrative divisions, structured 
Table 1 Relevant data and comparison among several countries

\begin{tabular}{llllll}
\hline Indicator & Benin & UK & Italy & Europe & USA \\
\hline Area $\left(\mathrm{km}^{2}\right)$ & 114,763 & 242,495 & 301,340 & $10,180,000$ & $9,833,520$ \\
Median age (years) & 18 & 40 & 45.9 & 42.8 & 38 \\
Number of physicians per 1000 people & 0.15 & 2.83 & 4.02 & 3.56 & 2.57 \\
GDP per person (\$) & 783.9 & 40,412 & $30,668.9$ & 35,632 & $52,194.9$ \\
\hline
\end{tabular}

in 34 sanitary zones. Beninese health system is structured in three levels, central (Ministry of Health and National Referral Hospital), intermediate (Departmental Referral Hospitals) and peripheral (Health zones, Commune Health Centers, village health units and private hospitals) [5], with significant diversities among different administrative divisions (e.g., north significantly different from south, and recently affected by hemorrhagic fevers cases).

During the Sixtieth World Health Assembly (May 2007), the World Health Organization (WHO) voted and adopted the Resolution WHA60.29 of 2007. As a primary result of this, WHO has recognized the severe implications of inefficient management of medical technology on the supply and quality of care in lower-income countries [6]. Three years later, in 2010, WHO published a report of its first international forum on medical devices [7], in which forty-two recommendations, regarding the safe use of medical devices, health technology assessment (HTA), the management and regulation of health technologies, were listed. Only 15 recommendations were prioritized, requiring $\mathrm{MoH}$ to adopt them within 2011 [7]. Specifically, to accomplish the final goal of these recommendations, i.e., to improve the operating practices of medical devices and equipment in hospitals, it is necessary to rely on data and information related to five aspects, namely the roles of medical devices, their availability, evaluation, management, and regulation. This data and information require a Computerized Maintenance Management System (CMMS) tool.

The use of medical devices varies with different medical interventions. Medical devices safety, effectiveness and efficiency depend on its complex and dynamic interaction with medical location context, which includes, but is not limited to [8]: patients, practitioners (i.e. medical-device User), combined use of device with drugs/food intake, interaction with other medical devices, accessories and consumables and medical location structures, plants and organization [9]. Therefore, it is crucial to contextualize them in such a complex context (Fig. 1), before designing a CMMS. While this may seem trivial in high resource settings, it is still an issue in lowresource ones such as Benin. Figure 1 is then essential to show our holistic approach that takes into account all entities surrounding the medical device. The considerations coming from this also support the registration fields of the proposed CMMS tool.
According to Gaev et al. [8], mastering the context requires a system approach and at least the following four interfaces, shown in Fig. 1, (respectively by lines $I_{1}, I_{2}, I_{3}$ and $I_{4}$ ) should be considered: Medical Device - User; Medical Device Patient; Medical Device - Environment; Medical Device Accessories. The latter is a generic representation of hospitals, regardless of any possible different implementation of it. According to the American College of Clinical Engineering (ACCE) and the Canadian Medical and Biological Engineering Society (CMBES) [10-12], experts in charge of supporting and advising medical staff in patient care (the user at the level of the $I_{1}$ interface) should master a considerable variety of engineering, organizational and management skills and offer training/education to medical healthcare. Examples of these skills are project management, health technology assessment, health technology management, risk management and standards compliance.

Following WHO Resolution WHA60.29, the Association for the Advancement of Medical Instrumentation (AAMI) published a report on the interoperability of medical devices in hospitals [13], in which it identifies and lists seven major pathways for the successful and safe operation of medical devices. They aimed to standardize, to motivate users with key roles and responsibilities, to promote patient safety and to streamline the clinical workflow. Figure 1 is a synthesis of these pathways for the safe operation of medical devices, according to the recommendations of ACCE, CMBES, WHO, AAMI. In particular, the resulting seven tracks were:

- $\quad P_{1}$ - the role of medical devices to improve the delivery of health services;

- $\quad P_{2}$ - safe, accessible and affordable medical devices: the availability of medical devices;

- $\quad P_{3}$ - health technology assessment;

- $\quad P_{4}$ - health technology management;

- $\quad P_{5}$ - regulation of health technologies;

- $\quad P_{6}$ - focusing first on human behavior;

- $\quad P_{7}$ - removing barriers through shared and continuous learning.

However, there is a need for prioritization for easier control of the situation by developing countries hospitals. Thus, we retain $P_{4}$ that leads to $P_{3}$ and $P_{6}$. In fact, in the eighties, higher income countries have made significant progress in the 
Fig. 1 Is inspired by [8] and it aims to represent how complex the ecosystem surrounding a medical device can be, and the huge number of factors, functions and actors that a designer should consider while designing a CMMS

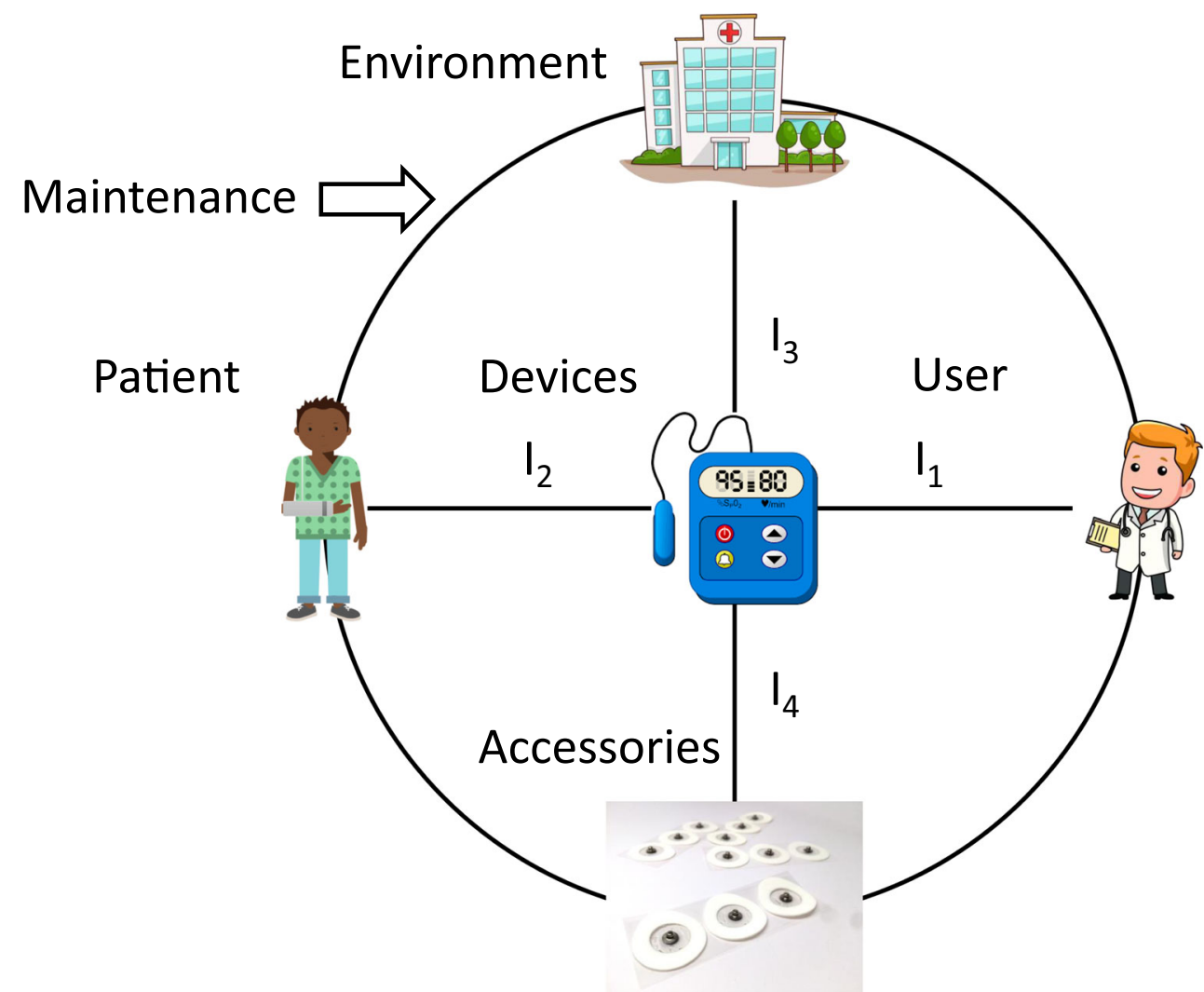

management of medical devices thanks to the integration of several technical experts [8] and the good practices of Biomedical Engineering in the context of Fig. 1. The latter ensures patient safety by the fact that the actors are well informed about medical devices, medical locations, their users and the surrounding cultural context.

As above-mentioned, health technology management and health technology assessment are two areas that need to be explored in the health systems of lower income countries. However, since traditional methods of health technology assessment may be difficult to apply to medical devices, both HTA and HTM have a clear need for reliable and updated information regarding medical devices, which requires the adoption of a CMMS. Moreover, as stated by Taktak et al. in [14] and Saranummi et al. in [15], the information arising from HTM and HTA, and the interactions among HTM, HTA and clinical personnel is one of the leading drivers for changes in hospitals. Information systems are used to support management and healthcare processes, which are two views to the patient care process that, despite what is commonly thought, complement each other [15].

In particular, all the technical, economic, structural, managerial efforts supporting clinical practice is referred to as "process of care". The latter is very complex as it requires interactions among several professionals (e.g. clinicians, clinical engineers, hospital engineers, hospital managers, nurses, IT technicians et cetera) and generates a huge amount of information regarding the status of medical locations (e.g., number of surgery deliverable in one day), of medical devices (e.g., serviced, functioning, sterilized if required, costs, consumables, staff training et cetera), of medical equips and patient timings et cetera Indeed, the process of care can be modeled as a non-linear system, since its response does not only depend on the input (e.g., patient severity, available medical devices, consumables, water, et cetera), but also on the status variable (e.g., medical location status, medical device functionality, personnel availability). Consequently, the management and evaluation of maintenance-oriented medical devices are based on a range of technical, clinical, scientific and managerial information for which hospitals across biomedical engineering departments use tools, such as CMMS [16, 17].

Regarding this topic, the afore-mentioned WHO resolution WHA60.29 requires the implementation of a CMMS tool that aims to « collect, verify, update and exchange information on medical technologies including particular medical devices to prioritize needs and allocate resources». Specifically, this information changes with the dynamism of the hospital and finds its main origin in the Equipment Park Inventory (EPI) that is an inventory of the sets of medical devices available in a hospital. As stated in $[18,19]$, the EPI must now be integrated into a CMMS tool to generate the information needed to implement the recommendations of [7], which will improve the overall management of medical equipment in a hospital [20]. Always according to WHO [20], the information contained in a CMMS system varies according to the local situation. Hence, a CMMS tool should be designed, developed, 
deployed and operated in each health system and hospital very cautiously.

Unfortunately, the fact that the USA, Europe and Japan represent the $80 \%$ of the medical device market [21], create a standard de facto [9]. Consequently, many CMMS adopt as 'reference' medical devices in developed countries (Fig. 2). Conversely, biomedical engineers should make an effort to design and develop CMMS that can be effectively deployed and operated in lower income countries. Figure 2 proposes a model aiming to illustrate how CMMS should be designed and deployed by measuring, analyzing and planning, relying on real data on medical devices in LICs. In particular, HTM and applied while designing such tools, which should take into account also the variety of contextual conditions of LICs. Such an effective CMMS is supposed to produce reports (outputs) that can help an efficient HTM and decisionmaking about health technologies.

There is extensive evidence proving that, although CMMSs are indispensable for the management of medical devices and medical locations [22, 23], sometimes their inputs and outputs have been the causes of their failures, also in higher income countries. The developed CMMS, then, should be validated during hospital trials in which important data can be collected and can be used to modify further and refine the prototype.

Moreover, when designing/implementing a CMMS tool for developing countries, it is crucial to use heuristics to analyze tries. Some elements could be (in bold the most limiting ones):

- limited budget vs high commercial costs;

- medical devices are designed to be operationalized in rich countries ( $80 \%$ of the market in the USA, Europe, Japan), where regulations are generally clear and presumably respected, therefore not resilient for all the low-income settings;

- chronic lack of specialized healthcare staff;

- chronic lack of specialized personnel for maintenance of medical devices and location;

- severe problems in the supply chain;

- heterogeneous software and devices coming from different manufacturers [14];

- limited availability of open source programs; international principles and best practices should be controlled systematically recurrent problems affecting lower-income coun-

- in the absence of strong local regulations and control functions, hospitals are often designed by international companies that may follow a significantly different standard (e.g., gas color code, which is different in Europe and China);

- a limited ecological barrier to medical device disposal;

- a significant number of medical devices are donated, generating a limited sense of responsibility for its maintenance;

- limited internet access and unreliable LANs

Although not exhaustive, this list of factors aims to present few essential elements that any designer of CMMS and healthcare technologies is supposed to familiarise with, before designing/deploying any CMMS or healthcare technology in a low-income setting. Also, even if some free software exists, these programs do not take into account the context of developing countries and access to such software requires a reliable internet connection that is not always available in such geographical areas [19] and specific CMMS tools for developing countries are still required [19].

This paper presents a successful case study, describing a CMMS tool designed by the Department of Biomedical Engineering of Ecole Polytechnique of Abomey Calavi (EPAC) of Benin and deployed in three Beninese hospitals. Such a tool was designed following the above-mentioned process of adapting to international standards and good practices and contextualizing it in the reality of a low-resource setting, namely Benin. Such tool will have a great impact on the maintenance and management of medical devices in the hospitals where it will be used and can be used as a reference by other researchers who decide to develop similar tools.

\section{Materials and methods}

\subsection{Key components of CMMS}

Starting from Figure 1, four CMMS sources of data were hypothesized: $I_{1}$ (Medical Device-User), $I_{2}$ (Medical DevicePatient), $I_{3}$ (Medical Device-Environment) and $I_{4}$ (Medical Device-Accessories) [8]. In addition, after analyzing the HTM model available in the three hospitals, three other complementary sources were added, namely: $I_{5}$ (Maintenance-Medical Device), $I_{6}$ (Maintenance-Accessories and Consumables) and $I_{7}$

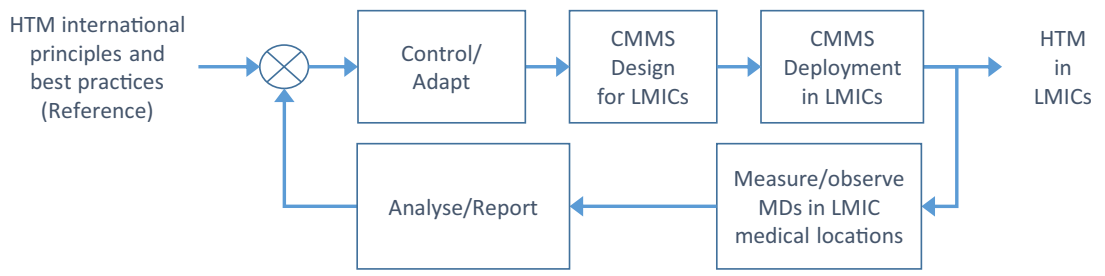

Fig. 2 Optimized design and deployment of CMMS in LICs. International principles and best practices of HTM are an essential reference. However, this reference requires control and adaptation, following a closed-loop control engineering approach, in which the feedback comes from reliable analyses and reports on real operational conditions of medical devices (MD) in LICs medical locations 
(Maintenance-Medical location). With respect to maintenance and maintenance management, fifteen interfaces were identified, together with their corresponding inputs.

\subsection{Input/output of CMMS}

The following steps were followed during the design of the CMMS, to capture properly all the processes that the CMMS should have supported:

- Identification and contextualization of the problems in two hospitals in Benin, one in the south and one in the north of the country;

- Cultural analysis of the environment with regard to Figure 1 in two hospitals, which allowed to obtain two promising tracks towards a CMMS tool for three months;

- Phase of design and realization of a model of the tool, based on the two hospitals;

- Design of an input data collection form according to the context of Benin health system;

- Identification of three hospitals, one in the north, one in the center and one in the south of Benin;

- Simultaneous inventory survey of three months in the three identified hospitals (at the rate of two persons per hospital), with the input data collection form followed by the on-site insertion of inputs in the CMMS tool;

- A feedback session consisting in a presentation workshop during which the CMMS design was presented and discussed to maintenance stakeholders (i.e., clinical/ biomedical engineers and technicians) including the maintenance services of the deployment hospitals in Benin;

- Development at the Laboratory of the Department of Biomedical Engineering of the EPAC. The tool was tested, verified and validated on the basis of the 2255 completed pen-and-paper forms for the inventories (see Figure 3). Figure 3, in particular, illustrates the first step of setting up a CMMS tool in a hospital in Benin. This is a good example to show the magnitude of such task: in fact, it requires going through inventories scanning for the great amount of existing equipment. This step is not to be underestimated as it is a preliminary result and is the basis of further work presented in the text.

\section{Results}

\subsection{GMaint-KM Benin: implementation}

A CMMS tool was developed and named "GMaint-KM Bénin". For the first version of the tool, 35 fields were included in the database, although such fields should be tailored to the reality of each hospital, as this is what the realities of low-resource settings require. In particular, the fields that were selected are either usually recommended for similar tools [20] ("Recommended" in Table 2) or draw on our experience and the realities of hospitals in Benin. Table 2 shows all the fields along with their justification. Moreover, as per WHO 2017 report, a National nomenclature of medical devices is absent in Benin. Therefore, the French one was adopted.

Afterward, five actors, responsible for the maintenance and management of medical equipment and devices, were identified for each hospital. Namely, the Director, the Head of the Department of Economic and Administrative Affairs (Chef du Service des Affaires Administratives Economiques (CSAAE)), the Chief Financial Officer (Chef du Service des Affaires Financiers (CSAF)), the Users, the Maintenance Service Manager, and the Maintenance technicians. In order to enrich the database, seven pen-and-paper registers (as shown in Figure 3) of the inventory of all medical and
Fig. 3 Illustration of the seven pen-and-paper registers obtained following the inventory campaign in the three selected hospitals
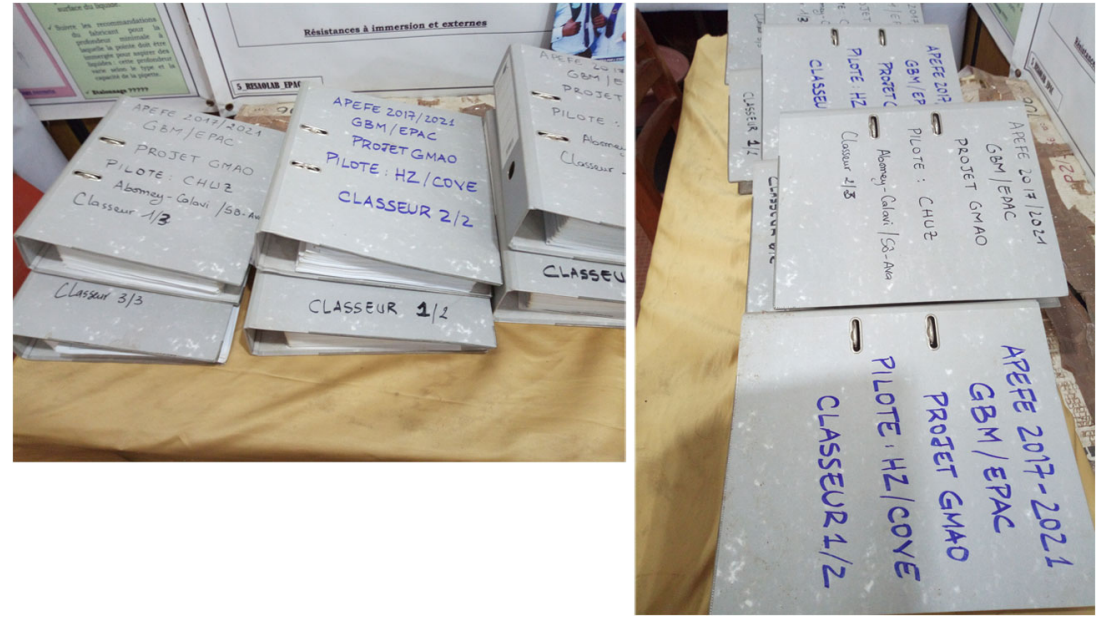
Table 2 The 35 fields included in the database along with their justification

\begin{tabular}{|c|c|c|}
\hline $\mathrm{N}^{\circ}$ & Field Name & Justification of the fields \\
\hline 1 & Funding (source) & To estimate the diversity of funding sources for equipment in a hospital \\
\hline 2 & Year of manufacture & Recommended \\
\hline 3 & Inventory number & Recommended \\
\hline 4 & External identifier & $\begin{array}{l}\text { A number available in the inventory of the hospital to identify the contractor who services/leases the devices available in the } \\
\text { hospital. }\end{array}$ \\
\hline 5 & Manufacturer & Recommended \\
\hline 6 & Mark & Recommended \\
\hline 7 & Type/Model & Recommended \\
\hline 8 & Serial number & Recommended \\
\hline 9 & Year of acquisition & Recommended \\
\hline 10 & Type of acquisition & To estimate acquisitions of new or used equipment in the form of: Donation, Purchase, Provision or Loan. \\
\hline 11 & Under warranty & Recommended \\
\hline 12 & Warranty duration & Recommended \\
\hline 13 & Warranty end date & Recommended \\
\hline 14 & Status & $\begin{array}{l}\text { To estimate at a given moment, the status of the equipment, namely: "functional" or "non-functional". If "non-functional", } \\
\text { the cause can be: "faulty reagent", or "maintenance failure", or "missing parts", or "lack of use", or "lack of consumables } \\
\text { or accessories" }\end{array}$ \\
\hline 15 & Volet (Shutter) & Defines if the equipment is biomedical or technical \\
\hline 16 & $\begin{array}{l}\text { Class Function } \\
\text { Equipment }\end{array}$ & Recommended \\
\hline 17 & Function & To know which care profile the equipment serves \\
\hline 18 & Service & Recommended \\
\hline 19 & $\mathrm{~N}^{\circ}$ Room & The room number of the equipment location \\
\hline 20 & Room & To register the service room where the equipment is located \\
\hline 21 & Mobility & To find out if the equipment is Static or Movable \\
\hline 22 & Supplier/Provider & Recommended \\
\hline 23 & Date of service & Recommended \\
\hline 24 & $\begin{array}{l}\text { Routine } \\
\quad \text { servicing/Upkeep }\end{array}$ & Recommended \\
\hline 25 & Periodicity & Recommended \\
\hline 26 & Main users/Owner & To specify if a device is dedicated to one user or shared among different wards \\
\hline 27 & Nature Category & To estimate the type of the equipment: Medical-technical device, Medical device, Device and conditioning, Related device \\
\hline 28 & Reason of acquisition & If the equipment is acquired for an update, replacement, or development \\
\hline 29 & User Manual & To estimate the percentage of equipment with user manuals \\
\hline 30 & Technical Manual & To estimate the percentage of equipment with technical manuals \\
\hline 31 & Commentary & Textbox used to add fundamental information about dedicated spare parts, special human resources, and related equipment. \\
\hline 32 & $\begin{array}{l}\text { Technical } \\
\text { Characteristics }\end{array}$ & To facilitate the strategies of electric safety tests \\
\hline 33 & CEE/EEC class & To facilitate the strategies of electric safety tests \\
\hline 34 & Electrical class & To facilitate the strategies of electric safety tests \\
\hline 35 & $\begin{array}{l}\text { Insulation/Isolation } \\
\text { Type }\end{array}$ & To facilitate the strategies of electric safety tests \\
\hline
\end{tabular}

medical-technical equipment were obtained for three selected hospitals, namely: The Saint Jean de Dieu Zone Hospital of Tanguiéta in northern Benin (H1); the Abomey-Calavi / SôAva Area Hospital and University Center in southern Benin (H2); the Agonlin Zone Hospital in Central Benin (H3). A total of 2255 items of equipment were inventoried, including 717 items from hospital H1, 924 items from hospital H2 and 614 items from hospital H3;
The paper inventories of each hospital were then compiled in the "GMaint-KM Bénin" tool and customized for each of the three hospitals (see Figure 3).

\subsection{GMaint-KM Benin: network configuration}

For each hospital, the personalized tool was tested, verified and validated in a local network at EPAC's Biomedical 
Fig. 4 The 2D configuration of local network deployment performed at EPAC's Laboratory of Biomedical Engineering Department

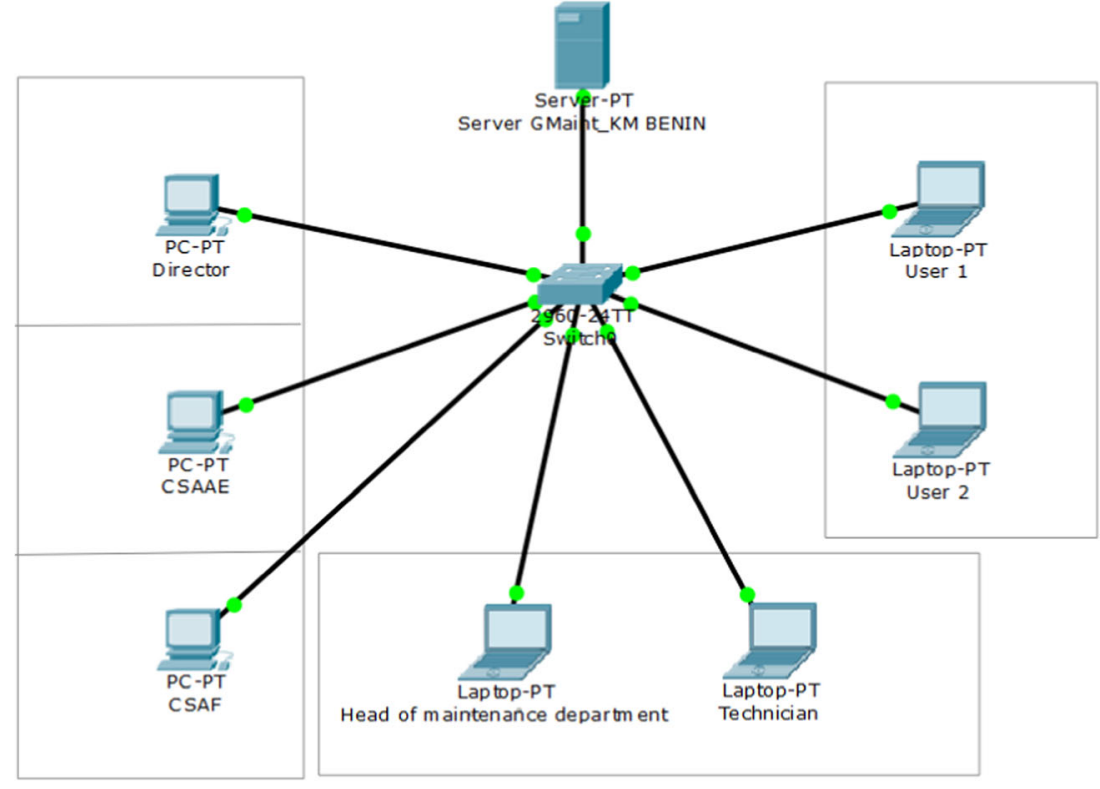

Engineering Laboratory. The configuration of the local network with the GSM option (Figure 4) was designed to consider the five main actors, who are concerned and involved in the management of maintenance, namely: The Director, the CSAAE, the CSAF, the Users, the Maintenance Service Manager, and Maintenance Technicians.

Fifteen categories of information and 69 direct outputs were finally identified. In order to manage such a variety of information, in each network, and therefore for each hospital, the "GMaint-KM Benin" tool was offered a set of filters allowing to display the 69 direct outputs (see Figures $5 \mathrm{a}-\mathrm{b}-\mathrm{c}$ and 6). The information provided by the outputs regards the five main actors in the maintenance management of equipment and devices in each hospital, according to the organization chart in hospitals in Benin.

Nine indirect outputs (from out70 to out78) were also obtained. Indeed, with the GSM option integrated, the "GMaintKM Bénin" tool offers the possibility by SMS of:

- Out70 - Request for intervention: To receive an SMS alert immediately, coming from the "GMaint-KM Bénin" tool as soon as a user requires intervention.

- Out71 - Preventive maintenance: To receive a reminder SMS, coming from the "GMaint-KM Bénin" tool, on the eve of each scheduled preventive maintenance.

- Out72 - Contract: To receive an SMS reminder, from the "GMaint-KM Bénin" tool, every seven days when there are maintenance contracts, whose deadlines are in less than fourteen days.

- Out73 - Recipient: To receive an SMS reminder, coming from the "GMaint-KM Bénin" tool, every five days when there are external service appointments, whose deadlines arrive in less than thirty days.
- Out74 - Evaluation report: To receive each Saturday, an SMS reminder, coming from the "GMaint-KM Bénin" tool, on the update of the evaluation report of the activities of the week.

- Out75 - Request suffering Intervention: To receive each fifth of the month an SMS of alert coming from the "GMaintKM Bénin" tool, which indicates the interventions, whose deadlines have been exceeded by more than thirty days.

- Out76 -Technical characteristics of a device (Optional): Possibility of sending an SMS to ask the "GMaint-KM Bénin" tool the technical characteristics of a device and to receive in return an SMS, which contains the required characteristics.

- Out77 - Devices attached to equipment (Optional): Possibility of sending an SMS to request the "GMaintKM Bénin" tool the devices attached to given equipment and receive in return an SMS that contains the list of these devices.

- Out78 - Functional state of a device (Optional): Possibility of sending an SMS to ask to the "GMaintKM Bénin" tool the operating status of a device and receive an SMS that provides information on the operating status of the equipment.

A user manual for the "GMaint-KM Bénin" tool was produced too.

\subsection{Statistical analysis}

Some real data extracted from the deployed CMMS are reported in Figure 7a that shows one of the possible outputs for hospital H2. As can be seen, it allows keeping track of several statistics and numbers regarding a selected 
a

\begin{tabular}{|c|l|c|c|c|}
\hline \multicolumn{1}{|c|}{ Outputs } \\
\hline
\end{tabular}

b

\begin{tabular}{|l|l|c|c|c|}
\hline \multicolumn{1}{|c|}{ Outputs } \\
\hline
\end{tabular}

C

\begin{tabular}{|l|l|c|c|c|c|}
\hline \multicolumn{1}{|c|}{ Outputs } \\
\hline
\end{tabular}

Fig. 5 a Screenshot of the direct outputs from 1 to 19 of the « GMaint-KM Benin » tool; b Screenshot of direct outputs from 20 to 42 of the "GMaint-KM Bénin" tool; c Screenshot of direct outputs from 43 to 57 of the "GMaint-KM Bénin" tool 
Fig. 6 Screenshot of direct outputs from 58 to 69 of the "GMaint-KM Bénin" tool

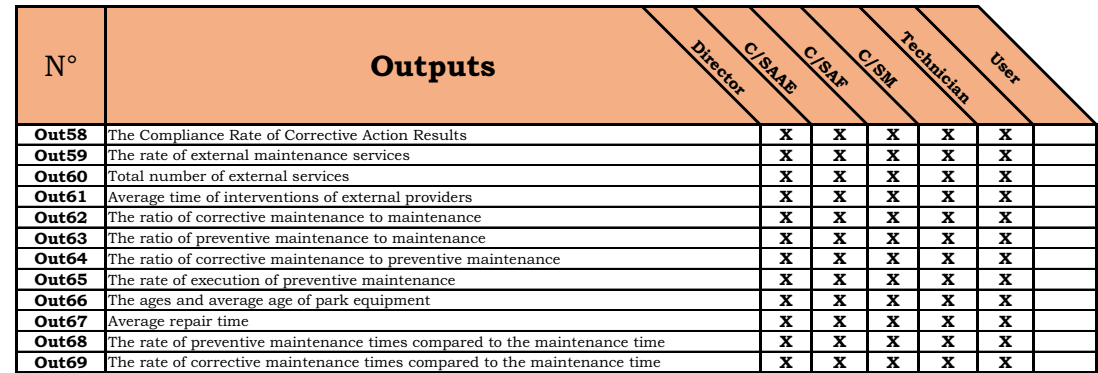

hospital. In this case, $68 \%$ of the information regarding the inventory data is obtained (924 medical and medicaltechnical equipment). For example, information about the year of manufacture, inventory number, model, year of acquisition, warranty, supplier, user manual and electric class etcetera can be retrieved. In this specific case, it can be seen that only $9.63 \%$ of medical devices hold information regarding the year of manufacture (i.e. 85 out of 924), no information about the manufacturer was available (0/924), only $2.92 \%$ of the devices was associated with warranty duration and warranty end date (5/924). Finally, only for less than $3 \%$ of the medical devices the electric class (34/924) was stated. On the other hand, there is abundant information (over 99\%) regarding the inventory number (917/924), the year of acquisition (922/924), if they are under warranty (924/924), the status (924/924) or user manual availability (924/924) et cetera.

Similarly, Figure $7 \mathrm{~b}$ shows the statistics regarding H3. In this case, around $73 \%$ of the information about the inventory data is obtained (614 medical and technical medical equipment). Like $\mathrm{H} 2, \mathrm{H} 3$ has not much information regarding the year of manufacture $(26.2 \%$ - 161/614), the manufacturer $(0 / 614)$ and warranty duration and end date $(0 / 614)$. On the other hand, much information $(100 \%)$ is retrieved regarding the year of acquisition (614/614), if the devices are under warranty (614/614), the status (614/614) or user manual availability (614/614) et cetera. Conversely to H2, H3 has more information as concerns the type/model $(86.9 \%$ - 534/614), the serial number $(72.3 \%$ - 444/614), the supplier $(100 \%$ $614 / 614)$, the technical characteristics $(90.5 \%$ - 556/614) and the electric class $(84 \%$ - 516/614).

To conclude the comparison, Figure $7 \mathrm{c}$ illustrates the statistics regarding $\mathrm{H} 1$ (717 medical and technical medical equipment), which has very similar trends to $\mathrm{H} 3$, apart from the information regarding the electric class (18\% - 129/717), which is significantly higher.

Other significant reports regarding the output include the difference between the year of manufacture and of acquisition and the average age of equipment per service. As an example, in $\mathrm{H} 2,7$ devices (8.8\%) are over five years old, with two of them being over 15 years old (Figure 8 ). In contrast, with mainly over alarmist literature, this data is in line with the European Coordination Committee of the Radiological
Electro-medical and Healthcare IT Industry (COCIR) golden rules. They suggest that only $30 \%$ of medical devices should be $6-10$ years old and only $10 \%$ of them should be over ten years old. However, COCIR states: "Medical technology more than ten years old is outdated and challenging to maintain and repair. Compared with current medical guidelines and best practices, it can be considered obsolete or inadequate for conducting some procedures; replacement is essential" [24].

Focusing on the average age of equipment per service (Figure 9), it can be seen that in $\mathrm{H} 2$ there are some obsolete (greater or equal to 6 years old) medical devices in the operating theatre, medical imaging, the laboratory, the morgue and the stomatology department. It is common sense that at least operating theatre and medical imaging services should have up-to-date technologies available, for a higher quality profile of care.

\section{Analysis and discussion}

Healthcare systems and medical locations in Sub-Saharan Africa are significantly different from those in the USA, Europe, and Japan, which represent the $80 \%$ of medical devices market, becoming a standard de facto for their design. HTM, as well as HTA, in Sub-Saharan Africa, require reliable and pragmatic experience of local conditions of medical devices and medical locations. In order to start managing this challenge, the Department of Biomedical Engineering of EPAC, in Benin, developed the CMMS tool "GMaint-KM Bénin". The design of the tool was very much need-driven and context-specific, although existing literature and international standards were considered as a reference during all the design process, using a system engineering closed-loop approach. Comparing the pen-and-paper registry and the CMMS presented, it is clear that the "GMaint-KM Benin" tool improved maintenance practices, making rapid failure analysis [17] possible and allowing prioritization of medical device interventions [25]. Those two functions were simply not possible on the pen-and-paper registry. It was not possible to make any comparison before/after the CMMS deployment, given the nature of the old registry. More detailed performance analysis will follow in the next months. 
Fig. 7 a Example of an output of the tool: inventory statistics for medical equipment at hospital $\mathrm{H} 2$; b Example of an output of the tool: inventory statistics for medical equipment at hospital $\mathrm{H} 3$; c Example of an output of the tool: inventory statistics for medical equipment at hospital $\mathrm{H} 1$
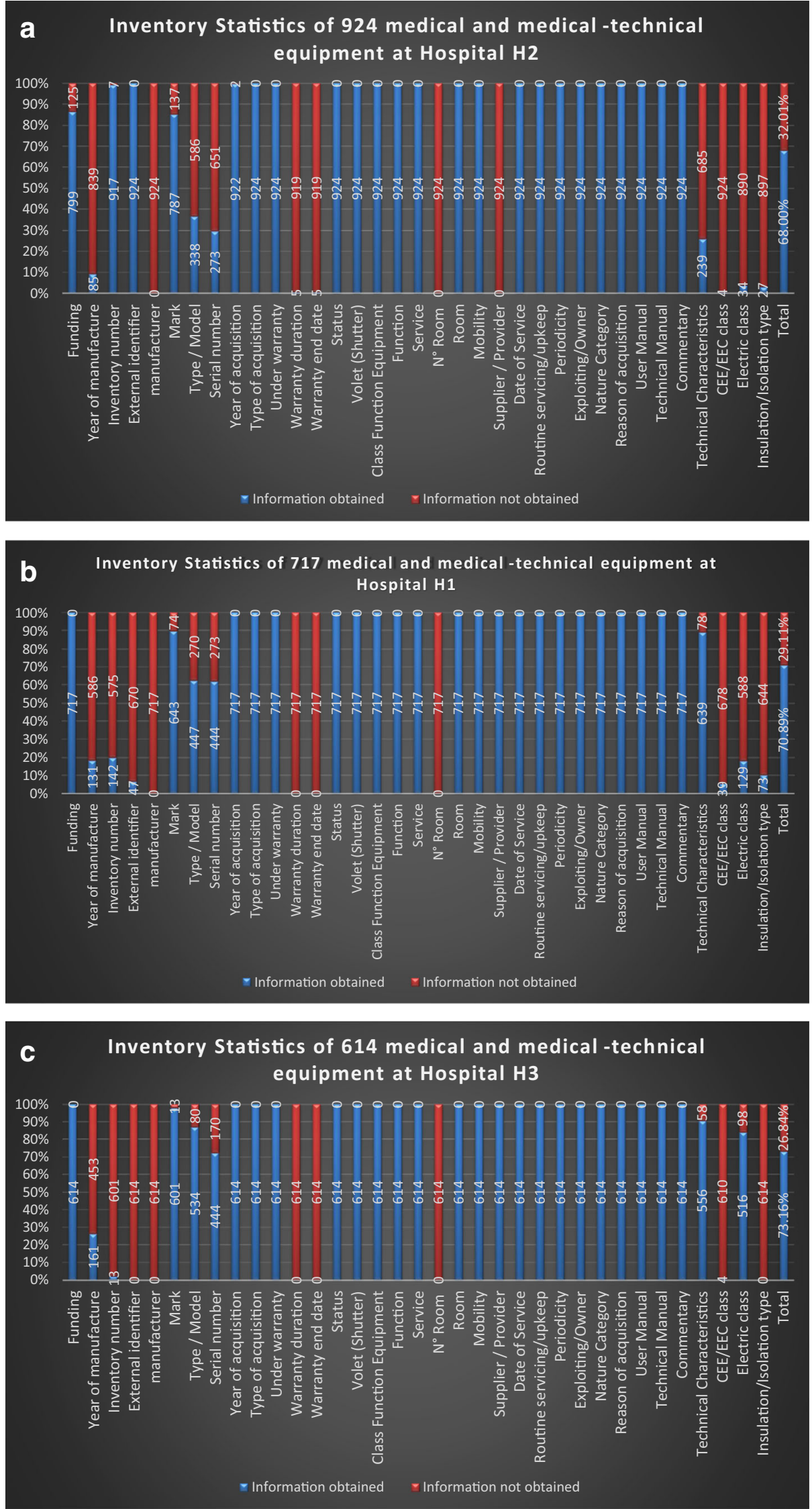


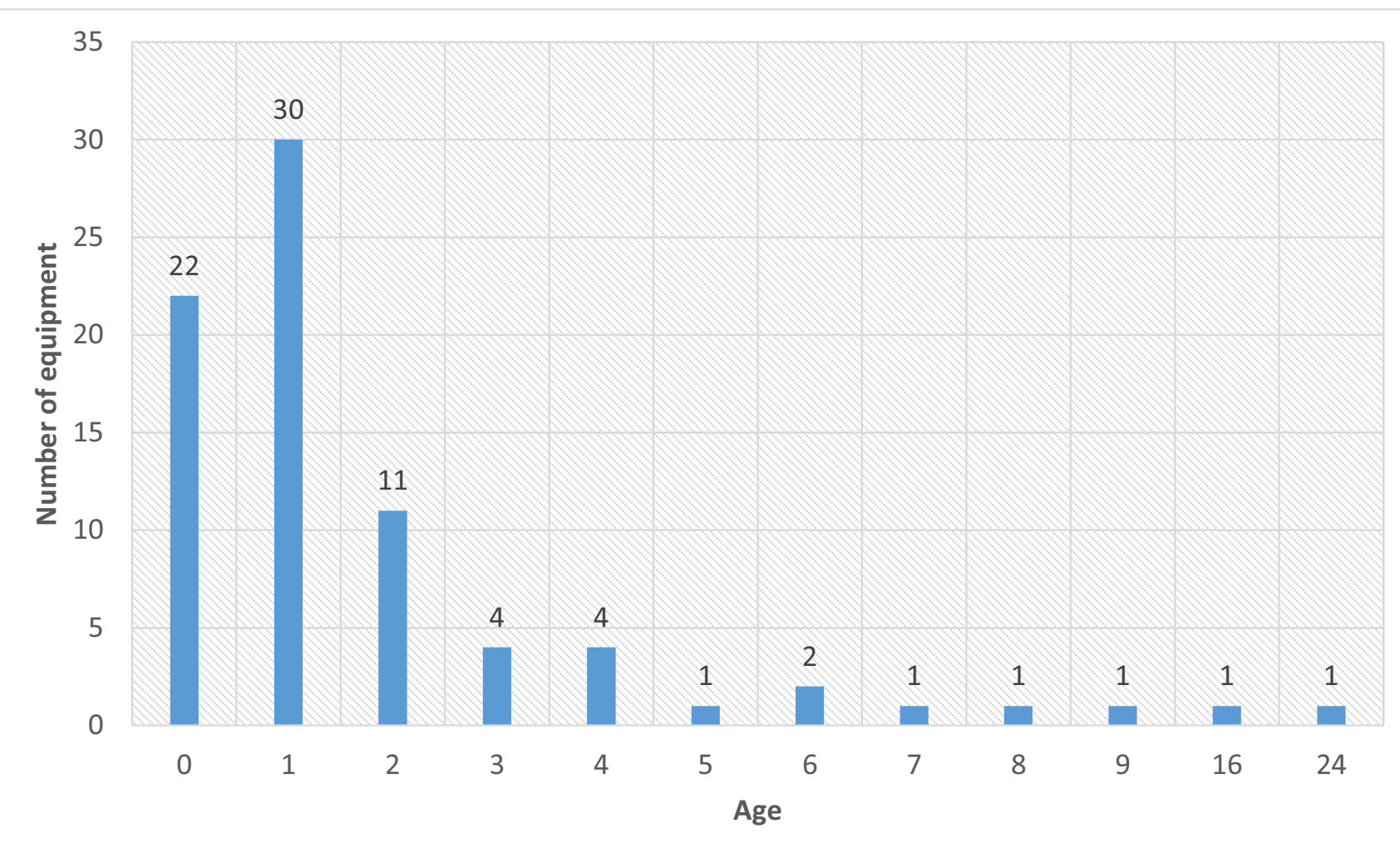

Fig. 8 The number of equipment per age group (difference between the year of manufacture and year of acquisition)

However, the tool is allowing to keep track of any type of tests and inspections performed during the life cycle of a medical device, such as acceptance testing, operational verifications, safety and performance inspections, and corrective maintenance actions [26]. The three hospitals were selected also considering the high quality of local technicians. However, the CMMS represents also a powerful tool for less experienced biomedical technicians, triggering their testing procedures. Finally, the current CMMS is driving appropriate medical devices acquisition, both via purchasing and donation.

One of the benefits of the CMMS lays in the actors' interaction [16]. Although the short lifespan of the CMMS does not allow quantitative performance measures, we observed
Fig. 9 The average age (years) of equipment per service

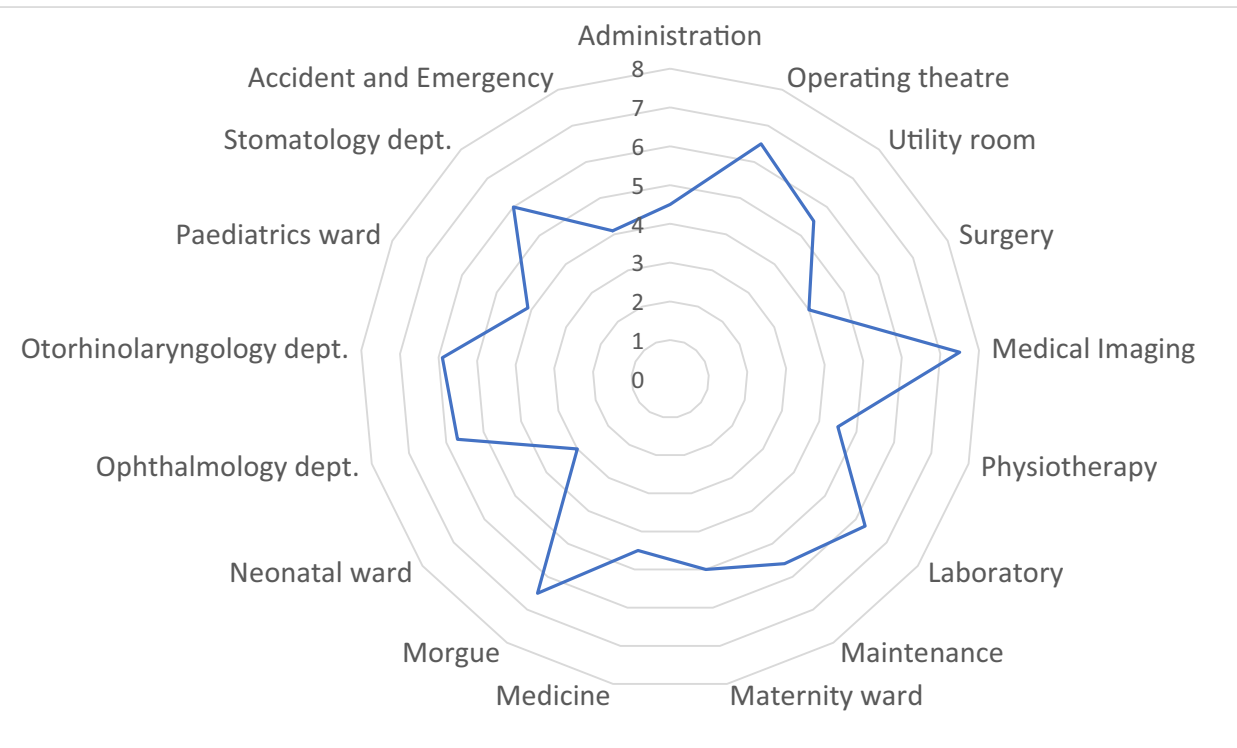


that the five key players identified in this project significantly improved their interaction, which was not functional and not traceable before the CMMS deployment. tThe results-outputs generated by the tool "GMaint-KM Bénin" were oriented and distributed to and on each of these main actors. A total of 78 outputs were generated (see Figures 5a-b-c and 6), 69 of which are direct outputs generated from the filters and interfaces of Figure 5 and nine indirect outputs generated for via the GSM option.

As expected from the core functions of a CMMS tool [26], "GMaint-KM Benin" allows controlling the inventory of the equipment park, dealing with the management of the work orders, controlling the management and the planning of the systematic preventive maintenance and the corrective maintenance, managing the suppliers and spare part provisioning. One limitation experienced during this project regarded the budgeting, that "GMaint-KM Benin" cannot currently support. However, the head of the budget session in the hospital can have access to it and receive useful data to budget for maintenance properly. A second limitation is that "GMaintKM Benin" tool currently semi-automatically manages the programming of routine preventive maintenance actions. Moreover, preventive maintenance and predictive preventive maintenance modules of the CMMS are not yet integrated as well as a checklist for routine actions and failure codes to facilitate reports to be generated. Those modules will be integrated only after the CMMS will have collected sufficient information in order to inform the tuning of preventive and predictive maintenance. Integrating those modules before collecting real data, would be against the spirit of this project and result, most likely in another ruinous topdown approach. At his moment, "GMaint-KM Benin" currently incorporates a single method for the prioritization criteria of the maintenance actions will be published elsewhere.

Overall, with "GMaint-KM Benin" and its 78 outputs, the data reach a well-defined channel in the biomedical engineering service, commonly called "biomedical maintenance" service in hospitals in developing countries. The tool considers the components of healthcare activity and is flexible so that it can satisfy potential needs that vary from one hospital to the other. For example control, measurement and test equipment can be entered in the tool "GMaint-KM Benin", so that this equipment can be maintained and documented with precision (see Figure 7a, b and c) [27].

Generally, three complications refrain the adoption of CMMS tools in lower-income country hospitals; namely, they are difficult to implement, they are expensive, and they are not available. "GMaint-KM Benin", overcomes these difficulties, verifies the recommendations of $[28,29]$ and follows an effective and appropriate need-driven and context-specific design model. Indeed, "GMaint-KM Benin" facilitates data entry, is flexible, configurable and performs error checks. This makes it possible to meet the needs of the different actors and, therefore, of different hospitals. The tool "GMaint-KM Benin" does a fairly complete task, which is to recall the story of what happened on a particular repair [29]. Moreover, it shows all the requirements of an ideal CMMS tool for the management of health technologies according to Cohen et al. [28], because it manages the data for the inventory and the monitoring of the equipment in the context of the specific characteristics of the equipment park in a health system of a developing country. In fact, "GMaint-KM Benin" manages and generates work orders, records the work done and generates hours of work. The innovation of this tool, opposite to WHO recommendations, is that unlike the INFRATECH project [19] that tries to discover open source CMMS software, it follows a systemic approach in the context of a hospital in a developing country. In fact, although open-access software can reduce initial costs, it still requires huge expertise for its installation, initialization and data entry. Certainly, in the majority of developed countries there is an abundance of experts to make this happen, but in lower income ones, it is not easy to find a sufficient number of well-trained technicians that can adapt, initialize and maintain open-software solutions.

In addition, the tool "GMaint-KM Benin" is adapted and adaptable, available and sustainable. The availability and sustainability are due to the fact that EPAC's Department of Biomedical Engineering is the only training center in Benin for biomedical technicians and engineers that trains cohorts of biomedical technicians for the labor market in the field. As it is in this department that the "GMaint-KM Benin" tool is developed with the support of all biomedical maintenance actors in hospitals in Benin, it will be systematically taught to students with the possibility of organizing recycle sessions for technicians currently in hospitals.

\section{Conclusion}

The design of the tool "GMaint-KM Benin" and its deployment in three hospitals in Benin, confirmed that especially in low-income settings, CMMS tools could significantly improve the management and control of the assets, allowing maintenance of medical devices and medical locations in hospitals. This paper illustrated the method employed, which consisted in using a system engineering closed-loop control approach, considering international standards and regulation as a required reference, but modulating the CMMS design and deployment with reliable information resulting from continuous monitoring of medical devices and medical location in the real context. Moreover, all the analysis and design was conducted by Beninese biomedical engineers, who are those having the best knowledge of real needs and context, which are required to lead such a successful project. The CMMS allowed bringing together the prerequisites that have not yet 
been fully met, regulatory compliance, financial performance and productivity monitoring of a park of medical devices and equipment in Benin. This CMMS will allow the Benin health system [30] to: standardize medical device incident reporting systems and processes, improve the integration of medical devices with information systems and clinical libraries, mitigate errors in medical devices use, significantly improve the management of medical devices and reconcile the challenges and differences in the environments of use of medical devices.

This project allowed pinpointing some critical differences regarding the management of medical devices among developed and developing countries. Most importantly, this project demonstrated that improvement in HTM in a very low-income country is possible when the design and the deployment are led by local experts and international experts limit their intervention to mentoring.

As future steps, in the short term, a mathematical model of the tool "GMaint-KM Benin" will be developed. Failure and repair codes will also be added in order to facilitate reports. Moreover, in its next version, the "GMaint-KM Benin" tool will be deployed at a national level in Benin, allowing the Ministry of Health to monitor the technical platform of the entire Benin health system. This is expected to strengthen measures for regulatory compliance and allows Benin to get closer to guidelines from ACCE, WHO and AAMI.

Eventually, in the medium and long-term, we will correct the tool according to real data rising from its utilization, although some actions are already planned, i.e. to integrate the budgeting module, to generate automatically the programming of the systematic, conditional and provisional preventive maintenance actions without forgetting the integration of a checklist for the actions of routines and several methods for establishing criteria for prioritizing maintenance actions.

Funding This project was realized with the financial support of the Association for the Promotion of Education Abroad (APEFE) in Benin. This is part of its multi-year program PPA 2017-2021 for which the Department of Biomedical Engineering of the EPAC has been selected. This is once again the place to express our gratitude to the APEFE Team in Benin. LP and DP received support from the University of Warwick with two Warwick Impact Found grants supported by the EPSRC Impact Accelerator Award (EP/K503848/1 and EP/R511808/1).

\section{Compliance with ethical standards}

Conflict of interest The authors declare that they have no conflict of interest.

Ethical approval This article does not contain any studies with human participants or animals performed by any of the authors.

Informed consent Not applicable.
Open Access This article is distributed under the terms of the Creative Commons Attribution 4.0 International License (http:// creativecommons.org/licenses/by/4.0/), which permits unrestricted use, distribution, and reproduction in any medium, provided you give appropriate credit to the original author(s) and the source, provide a link to the Creative Commons license, and indicate if changes were made.

\section{References}

1. World Population Review. 2018. http://worldpopulationreview. com/countries/benin-population/. Accessed 26/04/2018.

2. CIA. The world factbook. 2018. https://www.cia.gov/library/ publications/the-world-factbook/fields/2226.html. Accessed 26/ $04 / 2018$.

3. World Health Organization: The world health report 2006: working together for health. Geneva: World Health Organization; 2006.

4. World Bank Open Data. 2018. https://data.worldbank.org/. Accessed 26/04/2018.

5. Adeya G, Bigirimana A, Cavanaugh K, Franco LM. Rapid assessment of the health system in Benin: April 2006. Submitted to the US Agency for International Development. 2007.

6. WHO. The sixtieth world health assembly: Having considered the report on health technologies. WHA60. 29. 2007.

7. WHO. First WHO global forum on medical devices: context, outcomes, and future actions. 2011.

8. Gaev JA. Technology in health care. Clinical engineering handbook. Elsevier; 2004; 342-5.

9. Pecchia L. Health Technology Assessment of Medical Devices in Low and Middle Income Countries: study design and preliminary results. EMBEC \& NBC 2017. Springer. 2017; p. 225-8.

10. Kim SI, Suh TS. World congress of medical physics and biomedical engineering 2006: August 27-September 1, 20006 COEX Seoul, Korea. Springer Science \& Business Media. 2007.

11. ACCE. Clinical engineering and information technology. 2018. https://accenet.org/about/Documents/Clinical_Engineering_and Information Technology.pdf. Accessed 10-04-2018.

12. CMBES. CMBES Proclamation. 2018. https://www.cmbes.ca/ uploads/ck/files/clinical/cmbes_proclamation_2018.pdf. Accessed 10-04-2018.

13. Logan M, Patel B. Medical device interoperability: a safer path forward. Arlington: AAMI; 2012.

14. Taktak AFG, Paul G, David L, eds. Clinical engineering: a handbook for clinical and biomedical engineers. Cambridge: Elsevier Academic Press; 2013.

15. Saranummi N. Supporting system development with technology assessment. Assessment and Evaluation of Information Technologies in Medicine. Amsterdam: IOS Press; 1995. p. 35-44.

16. Fouad RH, Rawashdeh M, ALBashir A, Al-Sharif B. Designing a computerized maintenance management system for medical devices in royal medical services. 2012.

17. Osman HH, Hassan MA, Elhady NM, Elrasheed RM. Computerized preventive maintenance management system (CPMMS) for Haematology Department Equipments. International Journal of Computer Applications. 2015;109(16).

18. WHO. Introduction to medical equipment inventory management. 2011.

19. WHO. Second WHO global forum on medical devices: priority medical devices for universal health coverage. Geneva. 2013.

20. WHO. Computerized maintenance management system. 2011. $\mathrm{http} / /$ apps.who.int/medicinedocs/documents/s21567en/s21567en. pdf. Accessed 10-04-2018. 
21. Europe M. The European medical technology industry in figures. Brussels: MedTech Europe; 2013.

22. WHO. Programme de maintenance des équipements médicaux: présentation générale. 2012.

23. Santé Afdsdpd. Mise au point sur la Maintenance des Dispositifs Médicaux. 2011.

24. COCIR. Medical imaging equipment: age profile \& density. 2016.

25. Taghipour S, Banjevic D, Jardine AK. Prioritization of medical equipment for maintenance decisions. J Oper Res Soc. 2011;62(9):1666-87.

26. Taghipour S. Reliability and maintenance of medical devices: Citeseer. 2011.
27. Kinley CA. Healthcare Technology: A Strategic Approach to Medical Device Management: East Tennessee State University. 2012.

28. Cohen T, Cram N. Computerized maintenance management systems. J Clin Eng. 2001;26(3):200-11.

29. Stern G. Getting with the program to beef up cybersecurity. Biomed Instrum Technol. 2017;51(1):70-5.

30. Instrumentation AftAoM. Infusing patients safely: priority issues from the AAMI/FDA infusion device summit. AAMI/FDA Infusion Device Summit Proceedings. 2010:5-6.

Publisher's note Springer Nature remains neutral with regard to jurisdictional claims in published maps and institutional affiliations. 